\title{
Desequens: An $R$ package for the variance decomposition of sequential processes
}

\author{
Sofie Frederickx, Peter Kuppens, Francis Tuerlinckx, and Iven Van Mechelen \\ Katholieke Universiteit Leuven, Leuven, Belgium
}

\begin{abstract}
Recently, Kuppens, Van Mechelen, and Rijmen (2008) developed a method that allows researchers to examine and disentangle the contributions of the different possible sources of variability in sequential processes that underlie psychological outcomes or behaviors. Although this method may prove valuable for many research domains in the social sciences, its use may be limited by its statistical complexity and the effort and programming skills required. We present an $\mathrm{R}$ package, called Desequens, intended to make this method easily accessible to social science researchers. The tool does not require any knowledge of $\mathrm{R}$, so that $\mathrm{R}$ laymen can easily apply the method to their data as well. We demonstrate the use of Desequens by means of a didactic example.
\end{abstract}

Many psychological theories explicitly or implicitly rely on the assumption of sequential processes to explain outcomes or behavior. For instance, according to the theory of planned behavior, the display of a particular behavior depends on (1) how a situation evokes particular attitudes, subjective behavioral norms, and possibilities for behavioral control, and (2) how these variables, in turn, are related to the probability that the behavior will be displayed (Ajzen, 1991; see Van Coillie \& Van Mechelen, 2006, for an application to anger behavior). Similarly, the cognitive-affective personality system theory of Mischel and Shoda (1995) explains behavior by postulating that (1) situations activate cognitive-affective responses (such as goals, affects, etc.) that, in turn, (2) produce behavior, with individual differences characterizing the cognitiveaffective responses that are activated and what types of behaviors they elicit. For example, if another person bumps into you and spills coffee over your clothes, this may activate a hostile attribution unit, which in turn may lead to verbally aggressive behavior (e.g., scolding). Individual differences in aggressive behavior in such a situation then may result from individual differences in the linkage between the situation and hostile attribution and/or from individual differences in the linkage between hostile attribution and aggression. Note that in this case, one may wish to set up an intervention to reduce undesired aggression in this or similar situations. A correct identification of the locus of the most important individual differences in the sequential process underlying aggression may be of key importance.

Essential to these theories is that an outcome is assumed to result from a sequential psychological process in which (1) a situation elicits a number of mediating variables in a person and (2) these mediating variables, in their turn, elicit a certain target behavior. Figure 1 gives a graphi- cal representation of such a sequential process. Typically, the extent to which the mediating variables are elicited by a situation and how strongly they are related to the outcome depend on the person and therefore are subject to individual-difference variability. This implies that variability in the outcome is a function of variability in the different links of the hypothesized sequential processes. Information on the location and nature of the most important sources of variability in this sequential process is therefore crucial for the prediction and understanding of and intervention in the outcome of interest.

Traditional methods for studying mediation models (e.g., structural equation models) are not suitable for answering these questions. First of all, they do not leave room for individual differences. However, when the locus of the most important individual differences in sequential processes is studied, leaving room for individual differences is, of course, central. Second, they do not give direct estimates of the variance components.

Therefore, Kuppens, Van Mechelen, and Rijmen (2008) developed a method that allows researchers to examine and disentangle the contributions of the different possible sources of variability in the sequential processes that underlie psychological outcomes or behaviors. On the basis of data from different individuals on the mediating variables and the outcome in a set of different circumstances, the method yields a detailed picture of the relative importance of the different sequential sources of variability for the outcome of interest. For instance, suppose a researcher is interested in explaining eating behavior-more specifically, the amount of food intake during meals - and has collected information from different individuals on possible mediating variables of food intake (e.g., how hungry and attracted to the available food people were) in a number of circumstances and how much they eventually con- 


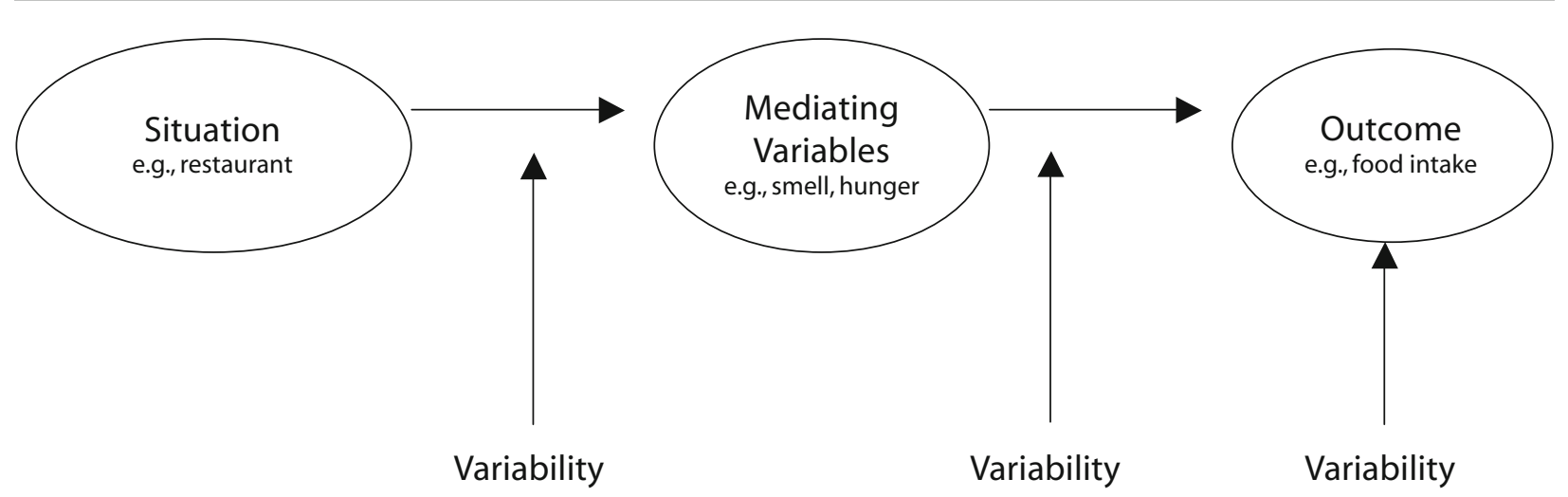

Figure 1. Graphical representation of the possible sources of variability in sequential psychological processes.

sumed in these circumstances (= outcome). On the basis of such data, the method developed by Kuppens et al. provides estimates of how much of the observed variability in food intake is due to variability in (1) how hungry and attracted to food people were in the various circumstances, which is further broken down into the separate contributions of individual differences, situational differences, and person $\times$ situation interaction plus error; (2) individual differences in how much hunger and attraction to food determine food intake; and (3) the part that cannot be explained by the hypothesized mediating process (e.g., differences in food intake that are not explained by the hunger and food attractiveness). In other words, it shows the researcher the extent to which how much we eat is due to variability in how hungry and attracted to food we are, to variability in how much these factors can determine our eating, and to individual-difference factors that the researcher has not considered so far. It is clear that this is valuable information for understanding the processes underlying eating behavior and for identifying the factors that should be targeted in interventions aimed at altering people's eating behavior.

Yet the method involves a number of sophisticated analyses (i.e., a random effects MANOVA and a random effects regression analysis), which yield estimates that subsequently have to be combined in a nontrivial way to calculate the relative contributions of the different sources of variability. The required estimates and combination are not standardly available in programs such as SPSS and SAS, and tailored methods and programming skills are needed to obtain the desired output. This combination of statistical complexity and required efforts and skills may make it prohibitive for researchers to use the method. As a way out, in this article, we provide an easy-to-use tool (called the Desequens package) in the freely available computing environment R (R Development Core Team, 2007; see Appendix A for how to obtain and install R and Desequens) for automatically obtaining the results of the method. The tool does not require any knowledge of $\mathrm{R}$, so that $R$ laymen can easily apply the method to their data as well.

In what follows, we will first present the details of the model developed by Kuppens et al. (2008). Next, we will explain the use of the Desequens package by means of an illustrative example.

\section{Method}

Data. In order to answer the question regarding the relative importance of different sources of variability in sequential processes, two pieces of information are needed. First of all, information is needed on the scores of person $j(j=1, \ldots, J)$ in situation $i(i=1, \ldots, I)$ on mediating variable $a(a=1, \ldots, A)$ - that is, $x_{a i j}$. Second, information is needed for all persons in all situations for the target behavior under study - that is, $y_{i j}$. Note that this assumes that the data were collected according to a fully crossed (factorial) design.

Model. The method relies on a combination of a MANOVA of the mediator scores (for an introduction, see, e.g., Bray \& Maxwell, 1985) and a mixed (multilevel) regression model for the studied outcome (for introductions, see, e.g., Nezlek, 2001; Snijders \& Bosker, 1999). The random effects MANOVA model for the mediator variables decomposes the (co)variances in the mediator variables into situation, person, and situation $\times$ person plus error contributions:

$$
\begin{gathered}
x_{1 i j}=\mu_{1}+\alpha_{1 i}+V_{1 j}+\varepsilon_{1 i j}^{x} \\
\ldots \\
x_{a i j}=\mu_{a}+\alpha_{a i}+V_{a j}+\varepsilon_{a i j}^{x} \\
\ldots \\
x_{A i j}=\mu_{A}+\alpha_{A i}+V_{A j}+\varepsilon_{A i j}^{x},
\end{gathered}
$$

with

$$
\left\{\begin{array}{l}
V_{a j} \sim \operatorname{MVN}\left(0, \Sigma_{V}\right) \\
\varepsilon_{a i j}^{x} \sim \operatorname{MVN}\left(0, \Sigma_{\varepsilon}\right)
\end{array}\right.
$$

for all $a \mathrm{~s}, i \mathrm{~s}$, and $j \mathrm{~s}$, where $\mu_{a}$ represents the overall mean of mediator $a, \alpha_{a i}$ represents the contribution of situation $i$ to mediator $a, V_{a i}$ reflects the contribution of person $j$, and $\varepsilon_{a i j}^{x}$ reflects the contribution of the person $\times$ situation interaction plus error (for partitioning into variance components in MANOVA, see Bock, 1975). 
In the multilevel model, the studied outcome is predicted by the (grand mean centered) mediator variables:

$$
y_{i j}=\gamma_{\mathrm{oo}}+U_{0 j}+\sum_{a=1}^{A}\left(\gamma_{a} x_{a i j}+U_{a j} x_{a i j}\right)+\varepsilon_{i j}^{y},
$$

where $\varepsilon_{i j}^{y} \sim N\left(0, \sigma^{2}\right)$ and $\gamma_{o o}$ and $\gamma_{a}$ denote the fixed intercept and slope values, respectively; $U_{0 j}$ and $U_{a j}$ denote the random, person-specific (as appears from the $j$ subscript) intercept and slope values; and $\varepsilon_{i j}$ denotes the residual variance.

Estimation. Estimations of the contributions of the different sources of variability can be obtained by combining the estimates from the random effects MANOVA (Equation 1) and the multilevel model (Equation 2). Estimating the MANOVA model gives an estimation of the person main effects $\left(V_{a j}\right)$, the situation main effect $\left(\alpha_{a i}\right)$, and the person $\times$ situation contribution (plus error) $\left(\varepsilon_{a i j}^{x}\right)$ and their covariances. Estimating the multilevel model provides estimations of the $\gamma \mathrm{s}$ and the (co)variances of $U_{0 j}, U_{a j}$, and $\varepsilon_{i j}^{\gamma}$.

Making the standard assumption of independence between $\left(U_{0 j}, U_{a j}\right)$, on the one hand, and $\left(x_{a i j}\right)$, on the other hand, it can be shown that estimates from the two models can be used to decompose the total variance in $y$ as in Equation 3 (see below). This decomposition directly corresponds to the different sources of variability of interest. (1) The first term reflects the total contribution of variability in the mediating variables in response to the situations, which is further broken down into the contributions of individual differences (reflecting the persons main effect), of situational differences (reflecting the situation main effect), and of person $\times$ situation interaction plus error. (2) The second term reflects the contribution of variability in the link between the mediating variables and the outcome. (3) The third term reflects the contribution of variability outside the hypothesized sequential process. (4) The last term represents the portion of variance in the outcome unexplained by the model. These analyses and the combination of their estimates are automatically performed by the Desequens package, which we will now introduce.

\section{The Desequens Package}

Data. The data have to be saved in a text file, with tabs used to separate the columns. There is no specific directory where the data need to be saved. The data should be in concatenated form - that is, with person-situation combinations as rows and the variables as columns. The variables should contain a person indicator, a situation indicator, a dependent variable, and a set of mediator variables. The first line of the data file should contain the names

$\begin{array}{llllll}\text { Sit } & \text { Pp } & \text { Out come Med1 } & \text { Med2 } & \text { Med3 } \\ 1 & 1 & 6 & 6 & 4 & 6 \\ 2 & 1 & 7 & 5 & 5 & 7 \\ 3 & 1 & 4 & 5 & 4 & 5 \\ 4 & 1 & 3 & 4 & 1 & 1 \\ 5 & 1 & 5 & 6 & 3 & 2 \\ 6 & 1 & 5 & 6 & 1 & 5 \\ 7 & 1 & 6 & 6 & 4 & 6 \\ 8 & 1 & 5 & 5 & 5 & 6 \\ 9 & 1 & 5 & 6 & 2 & 6 \\ 10 & 1 & 4 & 4 & 1 & 3 \\ 11 & 1 & 4 & 4 & 3 & 4 \\ 12 & 1 & 3 & 3 & 1 & 6 \\ 13 & 1 & 4 & 5 & 1 & 6 \\ 14 & 1 & 4 & 5 & 1 & 5 \\ 15 & 1 & 2 & 3 & 3 & 4 \\ 16 & 1 & 7 & 7 & 1 & 6 \\ 17 & 1 & 7 & 7 & 6 & 6 \\ 18 & 1 & 5 & 5 & 1 & 3 \\ 19 & 1 & 4 & 6 & 2 & 6 \\ 20 & 1 & 4 & 5 & 5 & 5 \\ 21 & 1 & 4 & 3 & 3 & 4 \\ 22 & 1 & 5 & 6 & 6 & 6 \\ 23 & 1 & 4 & 5 & 5 & 5 \\ 24 & 1 & 4 & 5 & 4 & 5 \\ 1 & 2 & 5 & 4 & 3 & 7\end{array}$

Figure 2. A screenshot of the required data structure.

of the different variables. Since some of the procedures involved in Desequens cannot handle missing data, it is required to listwise delete missing data from the data set. Figure 2 gives an example of how the data should look. Also, Desequens can work with R data frames.

General use of the Desequens package. The most important function of the Desequens package is the Ds.variancedecomposition function, which reads the data (through the Ds.readdata function) and performs the analyses and calculations necessary to obtain the variance component estimates (through the Ds.analyze function). To execute this function, type "vardecomp $\leftarrow$ Ds.variancedecomposition()" and execute the command. This command will call the Ds.variancedecomposition function and will store the results of this function in an object called vardecomp (which can be given any other name), including the results of the MANOVA and the multilevel analysis ${ }^{1}$ and the estimations of the contributions of the different sources of variability. In order to read the data, the Ds.variancedecomposition function makes use of pop-up windows. Typing a name that does not appear on the first line of the data file will prompt the questions to be repeated until all entered names are correct.

For advanced users, it is also possible to read in the data without making use of such pop-up windows. In order to do so, you need to give the required data information

$$
\begin{aligned}
\operatorname{Var}\left(y_{i j}\right)= & \underbrace{\sum_{a=1}^{A} \sum_{b=1}^{A}\left\{\gamma_{a} \gamma_{b}\left[\operatorname{COV}\left(\alpha_{a i}, \alpha_{b i}\right)+\operatorname{COV}\left(V_{a j}, V_{b j}\right)+\operatorname{COV}\left(\varepsilon_{a i j}^{x}, \varepsilon_{b i j}^{x}\right)\right]\right\}}_{1} \\
& +\underbrace{\sum_{a=1}^{A} \sum_{b=1}^{A} \operatorname{COV}\left(U_{a j}, U_{b j}\right) \operatorname{COV}\left(x_{a i j}, x_{b i j}\right)}_{2}+\underbrace{\operatorname{Var}\left(U_{0 j}\right)}_{3}+\underbrace{\operatorname{Var}\left(\varepsilon_{i j}^{y}\right)}_{4}
\end{aligned}
$$


Data information:

Hean of the dependent variable:

[1] 5.059564

Standarddeviation of the dependent variable:

[1] 1.608325

Minimum of the dependent variable:

[1] 1

Iaximum of the dependent variable:

[1] 9

Overview of the first and the last part of the dataset:

amount person situation hunger smell taste sight stress

$\begin{array}{llllllllll}1 & 5 & 20 & 1 & 2.9630872 & 2.4446309 & 1.3431208 & 2.4219799 & -3.015940\end{array}$

$\begin{array}{lllllllll}2 & 5 & 20 & 2 & 2.9630872 & 0.4446309 & 2.3431208 & 2.4219799 & -3.015940\end{array}$

$\begin{array}{llllllllll}3 & 6 & 20 & 3 & 0.9630872 & 0.4446309 & -0.6568792 & 0.4219799 & -2.015940\end{array}$

$\begin{array}{llllllllll}4 & 7 & 20 & 4 & 0.9630872 & 0.4446309 & 0.3431208 & 0.4219799 & -3.015940\end{array}$

$\begin{array}{lllllllll}5 & 7 & 20 & 5 & 2.9630872 & 1.4446309 & 1.3431208 & 2.4219799 & -3.015940\end{array}$

$\begin{array}{llllllllll}6 & 5 & 20 & 6 & 2.9630872 & 0.4446309 & 0.3431208 & 0.4219799 & -3.015940\end{array}$

$1 \quad$ pleasur

2.8196309

$3-1.1803691$

$4-1.1803691$

50.8196309

$6-1.1803691$

\begin{tabular}{rrrrrrrr}
\multicolumn{2}{c}{ amount } & person situation & hunger & sme 11 & taste & sight \\
1187 & 5 & 1005 & 3 & 0.9630872 & -1.5553691 & -1.6568792 & -1.5780201 \\
1188 & 1 & 1005 & 4 & -5.0369128 & -5.5553691 & -3.6568792 & -1.5780201 \\
1189 & 3 & 1005 & 5 & -5.0369128 & 0.4446309 & 0.3431208 & -1.5780201 \\
1190 & 1 & 1005 & 6 & -5.0369128 & -3.5553691 & -3.6568792 & -1.5780201 \\
1191 & 7 & 1005 & 7 & 2.9630872 & 2.4446309 & 0.3431208 & 0.4219799 \\
1192 & 3 & 1005 & 8 & 0.9630872 & -5.5553691 & -0.6568792 & -3.5780201
\end{tabular}

$\begin{array}{llll}1188 & 2.984060 & -5.1803691\end{array}$

$11892.984060-3.1803691$

$1190 \quad 3.984060-3.1803691$

$1191-3.015940 \quad 2.8196309$

$11924.984060-1.1803691$

Results:

Contributions of the persons, situations and person by situation interaction to the variance in mediators:

$\begin{array}{lrrrr} & \text { Person } & \text { Situation Person x Situation interaction (+error) } \\ \text { hunger } & 1529.126 & 355.517 & 2923.733 \\ \text { sme11 } & 734.096 & 678.319 & 1861.931 \\ \text { taste } & 735.039 & 780.945 & 2058.680 \\ \text { sight } & 764.369 & 853.187 & 1931.188 \\ \text { stress } & 1345.322 & 2200.670 & 3200.705 \\ \text { pleasure } & 905.846 & 777.523 & 1826.852\end{array}$

Percentual contributions of the persons, situations and person by situation interaction to the variance in mediators:

$\begin{array}{lrrr} & \text { Person } & \text { Situation Person x Situation interaction } & \text { (terror) } \\ \text { hunger } & 31.801 & 7.394 & 60.805 \\ \text { sme11 } & 22.420 & 20.716 & 56.864 \\ \text { taste } & 20.562 & 21.847 & 57.591 \\ \text { sight } & 21.539 & 24.042 & 54.419 \\ \text { stress } & 19.940 & 32.618 & 47.441 \\ \text { pleasure } & 25.806 & 22.150 & 52.044\end{array}$

Estimation of the fixed effects of the mixed regression analysis:

$\begin{array}{lrrr} & \text { Estimate Std. error } & \mathrm{T} \text { value } \\ \text { intercept } & 5.081 & 0.052 & 98.236 \\ \text { hunger } & 0.026 & 0.029 & 0.896 \\ \text { sme11 } & 0.097 & 0.038 & 2.585 \\ \text { taste } & 0.188 & 0.041 & 4.627 \\ \text { sight } & 0.127 & 0.041 & 3.122 \\ \text { stress } & -0.072 & 0.022 & -3.318 \\ \text { pleasure } & 0.135 & 0.039 & 3.455\end{array}$

Contributions of the different sources of individual differences:

$\begin{array}{lrr} & \text { Estimation } & \text { Percentage } \\ \text { Situation-Hediating variables } & 0.881 & 33.025 \\ \text { situational difference } & 0.280 & 10.502 \\ \text { individual differences } & 0.201 & 7.527 \\ \text { interaction plus error } & 0.400 & 14.996 \\ \text { Mediating variables-outcome } & 0.528 & 19.794 \\ \text { Sequence independent } & 0.189 & 7.087 \\ \text { Residual } & 1.070 & 40.093\end{array}$

Figure 3. Output of the summary function. 


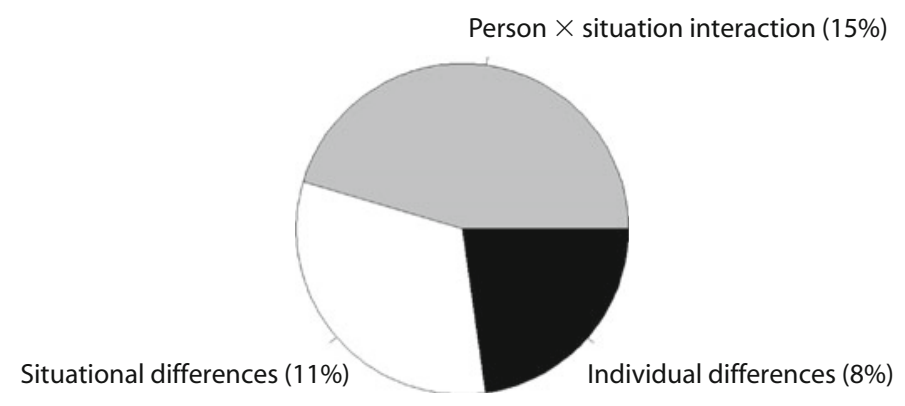

Figure 4. Pie chart of the contributions from persons, situations, and the person $\times$ situation interaction plus error to the variance in the studied mediating variables.

as arguments to the Ds.variancedecomposition function. More specifically, you need to type "vardecomp $\leftarrow$ Ds .variancedecomposition(popup = FALSE, data $=" \mathrm{C}: \backslash$ data.txt", DeV = "dependentvariable", Fperson = "personindicator", Fsituation = "situationindicator", predictors = c("medvar1", "medvar2"))." Note in this respect that your data can pertain either to an $\mathrm{R}$ data frame (i.e., data = data frame) or to an external text file (i.e., data = "C: \\data.txt"). The Ds.variancedecomposition function will then skip the Ds.readdata function and will immediately jump to the Ds.analyze function. For people without many programming skills, it is recommended to work with these pop-up windows, since this reduces the complexity greatly.

Once the analyses are finished, an overview of the results can be obtained by typing "summary(vardecomp)." This function yields four pieces of information. First, in order to be sure that you are working with the correct data set, the summary function gives some additional information about the data set (including the mean, standard deviation, minimum and maximum of the dependent variable, and the first part and the last part of the data set). Second, the contributions of the persons, situations, and the person $\times$ situation interaction to the variance in the mediating variables is given (absolute and in percentages.). Third, it shows the estimates of the fixed effects of the mixed regression analysis with associated standard errors and $t$ values. Fourth, it gives an overview of the estimations of the contributions of the different sources of variability, as outlined above.

Graphical presentations of the results are available through the Ds.piechart function. This function gives two pie charts - namely, a pie chart in which the contributions of the different sources of variability in the outcome are displayed and one in which the contributions of the persons, situations, and the person $\times$ situation interaction to the variance in the mediating variables are displayed. Type the command "Ds.piechart(vardecomp)" to produce the two pie charts.

Example analysis. We will illustrate the use of the Desequens package by analyzing data from a study on eating behavior (Vandeweyers, Ceulemans, \& Kuppens, 2008; these data are freely available in the "Data" subfolder of the Desequens package). In this study, in line with the personality theory of Mischel and Shoda (1995), data were collected to study cognitive-affective processes underlying eating behavior. Participants were asked to recall a total of eight different situations that were prespecified to include situations in which one was alone or not, in which one was under time pressure or not, and in which there was a large or small diversity of food available (yielding a total of eight possible combinations). On the basis of previous research on the determinants of eating behavior, the study included motivational (hunger), emotional (amount of experienced stress and pleasure), and sensory (attractiveness of smell, taste, and sight of the food) factors as potential cognitiveaffective mediating variables (Adam \& Epel, 2007; Maier, Vickers, \& Inman, 2007). After recalling each situation, the participants were asked to rate each situation with respect to all cognitive-affective variables and to indicate how much they ate in it, using 9-point scales (ranging from very little to very much). In principle, the variance decomposition as performed by Desequens assumes that the mediating

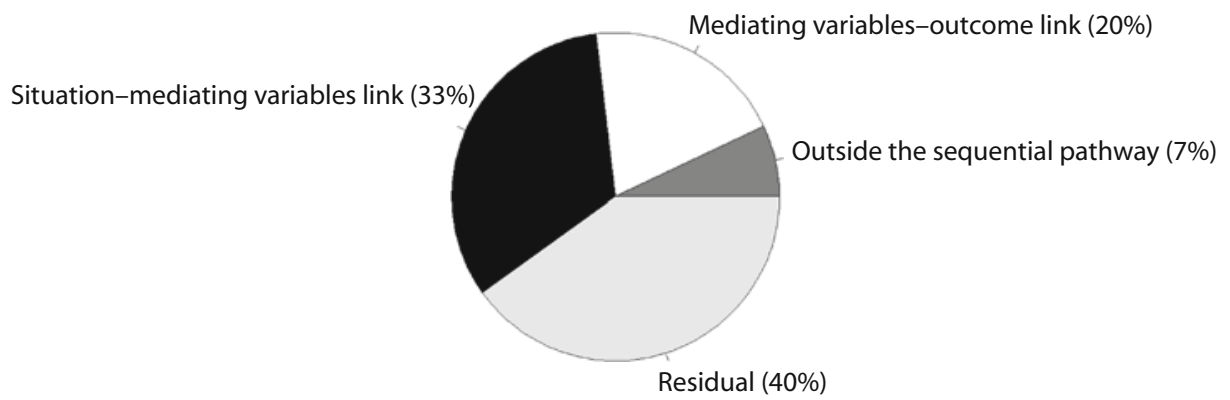

Figure 5. Pie chart of the contributions of the different sources of variability in eating behavior. 
and outcome variables are continuous (instead of ordinal scales, as in this example). However, we adopted the view of Tukey (1961/1986) and Velleman and Wilkinson (1993), who stated that the selection of statistical analysis methods based solely on the type of your measurement scale is inadequate. We also made use of a Likert scale with nine categories, which is a rather large number and for which it is common practice to consider such a scale as continuous.

To obtain estimates of the contributions of the different possible sources of variability in eating behavior, we first saved the data set in the appropriate form as "eatdata. txt". The commands we used are shown in Appendix B. We called the function Ds.variancedecomposition (Appendix $\mathrm{B}$ contains the codes both for when one wants to work with the pop-up windows and for when one wants to avoid them). To read the data, we typed the following in the subsequent pop-up windows: "C:Ivariancedecompositionleatdata.txt", "amount", "person", "situation", "taste sight smell stress hunger pleasure".

Applying the summary function gives us the information presented in Figure 3 in the R console, which gives an overview of all relevant information. The result of executing the Ds.piechart function is shown in Figures 4 and 5, which give a comprehensive overview of the variance components.

When the results are looked at, the MANOVA on the mediating variables showed that smell, sight, taste, and pleasure are almost equally determined by person and situation main effects (see Figure 3). Stress, on the other hand, is more situationally determined (stronger relative situation main effect), and hunger is more person related (stronger relative person main effect). The contribution of the interaction plus error is also substantial for the mediating variables. Second, the results from the mixed model regression analysis show that all mediating variables (except for hunger) contribute significantly to the prediction of how much people eat. Attractive smell, taste, and sight all increase the amount of food intake. Interestingly, of the emotional factors, the results indicate that when people experience stress, they tend to eat less, on average, but that when people feel good, they eat more. Third, the (relative) contributions of the different sources of variance show that the largest part of variability in eating behavior can be explained by variability in how people feel and are sensorially attracted to the food available in a particular situation (Figure 3 ). These aspects are roughly comparably determined by the person, the situation, and the interaction between both. Yet a large amount of the variability in eating behavior is also due to how important these factors are for different individuals in determining their eating behavior. Thus, how much people eat is determined not only by how stressed they are and how attracted they are to the food, but also, in part, by the idiosyncratic weights these variables have in determining the food intake in different people. The results further show that the theoretical model is fairly comprehensive, because systematic factors outside the hypothesized sequential process contribute much less to the total variance and the model captures $60 \%$ of the total variance in eating behavior.

\section{Conclusion}

In this article, we have presented an $\mathrm{R}$ package called Desequens, an easy-to-use tool for analyzing the contributions of the different sources of variability in sequential processes. We briefly described the method developed by Kuppens et al. (2008) and demonstrated the use of the Desequens package with a didactic example. We hope that Desequens will significantly lower the threshold for applying this method, making it amenable to researchers who are interested in examining the sources and nature of variability in hypothesized, sequential processes underlying behavior and other psychological phenomena.

\section{AUTHOR NOTE}

Correspondence concerning this article should be addressed to S. Frederickx, Department of Psychology, Katholieke Universiteit Leuven, Tiensestraat 102,3000 Leuven, Belgium (e-mail: sofie.frederickx@ psy.kuleuven.be)

\section{REFERENCES}

AdAM, T. C., \& Epel, E. S. (2007). Stress, eating and the reward system. Physiology \& Behavior, 91, 449-458.

Ajzen, I. (1991). The theory of planned behavior. Organizational Behavior \& Human Decision Processes, 50, 179-211.

BATES, D. (2005). Fitting linear models in R: Using the lme4 package. $R$ News, 5, 27-30.

Bates, D., Maechler, M., \& Dai, B. (2008). 1me4: Linear mixedeffects models using S4 classes (R package version 0.999375-27). http://lme4.r-forge.r-project.org.

Bock, R. D. (1975). Multivariate statistical methods in behavioral research. New York: McGraw-Hill.

Bray, J. H., \& MaXwell, S. E. (1985). Multivariate analysis of variance. (Sage University Paper Series on Quantitative Research Methods, Vol. 54). Newbury Park, CA: Sage.

Kuppens, P., Van Mechelen, I., \& Rijmen, F. (2008). Towards disentangling sources of individual differences in appraisal and anger. Journal of Personality, 76, 969-1000.

Maier, A., Vickers, Z., \& InMan, J. J. (2007). Sensory-specific satiety, its crossovers, and subsequent choice of potato chip flavors. Appetite, 49, 419-428.

Mischel, W., \& Shoda, Y. (1995). A cognitive-affective system theory of personality: Reconceptualizing situations, dispositions, dynamics, and invariance in personality structure. Psychological Review, 102, 246-268

NezleK, J. B. (2001). Multilevel random coefficient analyses of eventand interval-contingent data in social and personality psychology research. Personality \& Social Psychology Bulletin, 27, 771-785.

R Development Core Team (2007). $R$ : A language and environment for statistical computing. Vienna: R Foundation for Statistical Computing. Available at www.r-project.org.

SNiJders, T. A. B., \& Bosker, R. J. (1999). Multilevel analysis: An introduction to basic and advanced multilevel modeling. London: Sage.

TukeY, J. W. (1986). Data analysis and behavioral science or learning to bear the quantitative man's burden by shunning badmandments. In L. V. Jones (Ed.), The collected works of John W. Tukey (Vol. 3, pp. 391-484). Belmont, CA: Wadsworth. (Original work published 1961)

Van Coillie, H., \& Van Mechelen, I. (2006). Expected consequences of anger-related behaviors. European Journal of Personality, 20, 137-154.

Vandeweyers, I., Ceulemans, E., \& Kuppens, P. (2008). Determinants of eating behavior. Unpublished master's thesis, Katholieke Universiteit Leuven

Velleman, P. F., \& Wilkinson, L. (1993). Nominal, ordinal, interval, and ratio typologies are misleading. American Statistician, 47, 65-72. 


\section{NOTE}

1. In order to perform the multilevel analysis, we make use of the packages "Matrix" and "Ime4" (Bates, 2005; Bates, Maechler, \& Dai, 2008). These packages are automatically loaded by the
Ds.variancedecomposition function. However, if these packages are not part of one's "load packages" menu, one will have to load these packages oneself, making use of the install.packages(“") command. More specifically, we make use of the "REML" method to fit the multilevel model, which means that the goal is to maximize the restricted log-likelihood.

\section{APPENDIX A}

Availability Section

\section{Installation of $\mathbf{R}$}

Persons who already have $\mathrm{R}$ installed on their computer should make sure that they are working with the latest version of $\mathrm{R}$ (i.e., 2.8.0). If this is not the case, they should update their version of $\mathrm{R}$.

The $\mathrm{R}$ installation files are distributed by the Comprehensive $\mathrm{R}$ Archive Network (CRAN). CRAN is a collection of sites that contain identical materials and was created to decrease the load on any one server. To install $\mathrm{R}$, go to the cran.r-project.org/mirrors.html Web site. First of all, one must choose a close location. After doing this, one can choose in the "Download and Install R" box which version of R one wants to use: Mac OS X, Windows, or Linux.

$\mathrm{R}$ for Mac OS X is available on CRAN in the form of a disk image, "R.dmg with optional version number," containing a package consisting of the R framework, the R.app GUI, and optional libraries. If one double-clicks on the disk image, a new Window opens with the instellar package. With respect to the Windows version, one must click on the "base" link and then right-click on the ".exe file" link and choose "Save as." For the Linux version, one must choose the subdirectory that matches one's distribution until one finds an .rpm file, which one must install. More detailed information can be found at cran.r-project.org/doc/manuals/R-admin.html.

\section{Installation of Desequens}

In order to install the Desequens package, go to the "package" menu, choose "install packages from local zip files ..." and load the "Desequens_1.0.zip" file (which can be freely downloaded from ppw.kuleuven.be/okp/ software/desequens). Next, type library("Desequens") in the R script and execute this command. An R script (see File-New script, in the menu taskbar) is a text file containing the same commands that one would enter on the command line of the R console. Working with scripts, instead of typing everything immediately in the $\mathrm{R}$ console, has the advantage that commands can be changed and saved easily and quickly (hence, it comes close to the ideal of reproducible statistics, in which anyone is able to replicate the analyses). If one wants to execute the commands of the R script, one must select the commands and press Control + "R."

\section{APPENDIX B}

\#\# loading the Desequens package

library("Desequens")

\# reading the license

Ds.License ()

\#\# read in the data and perform the MANOVA and the multilevel analysis \#\# with pop-up windows

vardecomp $<$ - Ds.variancedecomposition() \#\# without pop-up windows

vardecomp < - Ds.variancedecomposition(popup = FALSE, data = eatdata, DeV = "amount", Fperson = "person",

Fsituation = "situation", predictors = c("hunger", "smell", "taste", "sight", "stress", "pleasure"))

\#\# getting an overview of the results

summary(vardecomp)

\#\# creating the pie charts

Ds.piechart(vardecomp)

(Manuscript received October 7, 2008

revision accepted for publication January 27, 2009.) 